\title{
Integração de sistemas de gerenciamento ERP para contribuição na gestão do
}

\section{conhecimento empresarial}

Valdemilson Araújo

Bacharel em Ciência da Computação; Pós-Graduado em Gerenciamento de Projetos; Mestrando em Administração - Gestão de Projetos - Universidade Nove de Julho- UNINOVE milsoncomputacao@gmail.com

Isabel Cristina Scafuto

Doutora em Administração na Universidade Nove de Julho. Professora e Pesquisadora no Programa de Pós-Graduação em Gestão de Projetos - PPGP da Universidade Nove de Julho - UNINOVE.

isabelscafuto@gmail.com

\section{Editor Científico: José Edson Lara}

Organização Comitê Científico

Double Blind Review pelo SEER/OJS

Recebido em 01.09.2019

Aprovado em 08.11.2019

\section{(c) (i) (9)}

Este trabalho foi licenciado com uma Licença Creative Commons - Atribuição - Não Comercial 3.0 Brasil 


\title{
Resumo
}

Objetivo do estudo: Entender como o gerenciamento de projetos contribuiu para a implementação e integração de dois sistemas ERP, com o intuito de melhorar a padronização da Gestão do Conhecimento dessas empresas.

Metodologia/abordagem: A abordagem utilizada para o estudo será de natureza qualitativa e, como método, será aplicado uma pesquisa de campo com o uso de entrevistas semiestruturadas. Também serão coletados dados secundários das empresas, como, documentos que fizeram parte do processo de implementação e, também o referencial teórico que dará suporte para as análises. Assim, será possível realizar a triangulação dos dados. Os dados, após serem coletados, serão analisados com o auxílio do software MAXQDA.

Originalidade/Relevância: $O$ estudo se justifica por apresentar uma aplicação prática da gestão de projetos na implementação do software ERP em duas empresas distintas e, uma confirmação empírica da relação entre os temas.

Principais resultados: Demonstram que a gestão de projetos usada de maneira profissional e não intuitiva colabora com um bom andamento do projeto. Ao contrário, usada sem rigor metodológico acarreta desordem no projeto. E que a implementação do sistema ERP para a gestão do conhecimento, corroborando com a literatura, realmente implica numa melhor estratégia proporcionando vantagem competitiva para a empresa.

Contribuições teóricas/metodológicas: $O$ estudo contribui com a relação da gestão do conhecimento e a gestão de projetos de implementação de software ERP que foram testadas empiricamente, indo ao encontro dos autores abordados.

Contribuições sociais para a gestão: Contribui como um guia das melhores práticas para os gestores de projetos de gestão de conhecimento.

Palavras-chave: Gestão de Projetos; Gestão do Conhecimento; Sistemas ERP.

\section{Integration of ERP management systems to contribute to enterprise knowledge management}

\begin{abstract}
Objective of the study: To understand how project management contributed to the implementation and integration of two ERP systems, in order to improve the standardization of Knowledge Management of these companies.

Methodology / approach: The approach used for the study will be qualitative in nature and, as a method, a field research will be applied using semi-structured interviews. Secondary data will also be collected from companies, such as documents that were part of the implementation process and also the theoretical framework that will support the analysis. Thus, it will be
\end{abstract}


possible to perform the triangulation of the data. The data, after being collected, will be analyzed with the aid of MAXQDA software.

Originality / Relevance: The study is justified by presenting a practical application of project management in the implementation of ERP software in two different companies and an empirical confirmation of the relationship between the themes.

Key Findings: Demonstrate that professional, non-intuitive project management collaborates with good project progress. On the contrary, used without methodological rigor causes disorder in the project. And that the implementation of the ERP system for knowledge management, corroborating the literature, really implies a better strategy providing competitive advantage for the company.

Theoretical / methodological contributions: The study contributes to the relationship between knowledge management and project management of ERP software implementation projects that were empirically tested, meeting the authors approached.

Social Contributions to Management: Contributes as a best practice guide for knowledge management project managers.

Keywords: Project Management; Knowledge management; ERP systems.

\section{Integración de sistemas de gestión ERP para contribuir a la gestión del conocimiento empresarial}

\section{Resúmen}

Objetivo del estudio: comprender cómo la gestión de proyectos contribuyó a la implementación e integración de dos sistemas ERP, con el fin de mejorar la estandarización de la gestión del conocimiento de estas empresas.

Metodología / enfoque: el enfoque utilizado para el estudio será de naturaleza cualitativa y, como método, se aplicará una investigación de campo mediante entrevistas semiestructuradas. Los datos secundarios también se recopilarán de las empresas, como los documentos que formaron parte del proceso de implementación y también el marco teórico que respaldará el análisis. Por lo tanto, será posible realizar la triangulación de los datos. Los datos, serán analizados con la ayuda del software MAXQDA.

Originalidad / Relevancia: El estudio se justifica presentando una aplicación práctica de gestión de proyectos en la implementación del software ERP en dos compañías diferentes y una confirmación empírica de la relación entre los temas.

Resultados clave: demuestre que la gestión de proyectos profesional y no intuitiva colabora con el buen progreso del proyecto. Por el contrario, el uso sin rigor metodológico provoca desorden en el proyecto. Y que la implementación del sistema ERP para la gestión del 
conocimiento, corroborando la literatura, realmente implica una mejor estrategia que proporciona una ventaja competitiva para la empresa.

Contribuciones teóricas / metodológicas: el estudio contribuye a la relación entre la gestión del conocimiento y la gestión de proyectos de proyectos de implementación de software ERP que se probaron empíricamente, reuniéndose con los autores abordados.

Contribuciones sociales a la gestión: Contribuye como una guía de mejores prácticas para los gerentes de proyectos de gestión del conocimiento.

Palabras clave: Gestión de proyectos; Gestión del conocimiento; Sistemas ERP.

\section{Introdução}

A informação tem sido fundamental para a sobrevivência das organizações. E os sistemas de informação, a cada dia, são desenvolvidos e implementados para auxiliar no gerenciamento eficiente do conhecimento corporativo (Xu, L.; Wang, C.; Luo, X. \& Shi, Z, 2006). Os sistemas de informação, como os sistemas (ERP) Enterprise Resource Planning são implementados de forma rápida para ajudar as empresas a melhorar sua competitividade (Xu et al., 2006).

Atualmente, muitas empresas de software desenvolvem um produto genérico e fazem modificações conforme as necessidades de cada cliente, é o caso de um sistema de planejamento de recursos empresariais (ERP) (Sommerville, 2007). O ERP é desenvolvido como um sistema grande, complexo e é adaptado a uma empresa, incorporando informações sobre as regras e os processos de negócios da empresa (Sommerville, 2007). E as organizações atuais administram seus negócios com auxílio da Gestão do Conhecimento, gerando certo poder de competitividade com seus conhecimentos em um determinado produto/serviço e inovações (Xu et al., 2006). Assim, os ERPs são ferramentas importantes para auxiliarem a Gestão de Conhecimento nas empresas. Além disso, cada vez mais as empresas estão utilizando atividades baseadas na Gestão de Projetos para melhorarem a sua competitividade (Bartsch; Ebers, \& Maurer, 2013).

Os fatos apresentados neste estudo relatam sobre uma empresa sediada em São Paulo (seu nome será mantido em sigilo), do setor de distribuição de papel, que sentiu a necessidade de implementar e integrar o sistema ERP com a abertura de outra empresa do mesmo grupo. Esta implementação e integração foi necessária para a padronização e melhoria da Gestão de Conhecimento da empresa. A empresa desenvolvedora do ERP fez todo o processo utilizando a Gestão de Projetos para conduzir o trabalho. 
Portanto, a proposta deste estudo é o de entender, por meio de uma pesquisa qualitativa com triangulação de dados, como o gerenciamento de projetos contribuiu para a implementação e integração de dois sistemas ERP em duas empresas distintas de um mesmo grupo, com o intuito de melhorar a padronização da Gestão do Conhecimento dessas empresas. Foram realizadas entrevistas com perguntas semiestruturadas aos envolvidos no projeto, além de consulta aos documentos do projeto. E, os dados foram analisados com a ajuda do software MAXQDA.

\section{Referencial Teórico}

\subsection{Gerenciamento de projetos}

Existe um crescimento no uso de projetos como forma de instituir mudanças nas organizações (Crawford e Hassner-Nahmias, 2010). Kerzner (2013) aborda sobre uso de gerenciamento de projetos no importante impacto da mudança organizacional e na iniciação, processamento e implementação de projetos. A elaboração de projetos está sendo a cada dia mais valorizado nas organizações modernas, principalmente para suas iniciativas de gestão estratégica de negócios. Um projeto é uma série de atividades / tarefas que têm um objetivo específico a ser completado dentrode uma data inicial e final; tem limites de financiamento e gastos, pessoas e equipamentos; e são multifuncionais (Kerzner, 2013).

A década de 1990 foi fortemente influenciada pelo assunto gerenciamento de projetos, sendo observada de duas formas (Carvalho \& Rabechini, 2013). A primeira tratando das questões básicas do gerenciamento de projetos como cursos de treinamento, observância na gestão do escopo, acompanhamentos de prazos / custos por meio de cronogramas, aumento da utilização da tecnologia da informação para obter informações precisas por meio de softwares desenvolvidos para a área de gerenciamento de projetos (Carvalho \& Rabechini, 2013). Com isto, proporcionou maior eficiência para as empresas no gerenciamento de projetos com o uso de técnicas e práticas para utilização dos recursos permitindo fazer as coisas corretamente (Tereso, et al. 2018). As empresas precisam de gerenciamento de projetos profissional, obter resultados mais rápidos, de forma eficaz (Carvalho \& Rabechini, 2013).

A segunda, além de cumprir com todos os aprendizados da primeira, deverá ser mais eficaz no gerenciamento de projetos na área organizacional (Carvalho \& Rabechini, 2013). Deverá se apresentar de forma profissional, com mais criatividade e sendo menos intuitiva, 
levando o gerenciamento de projeto como proposta de inovação das atividades gerenciais (Martens, et al., 2014). Deverá fazer uso da integração das áreas do conhecimento em gestão de projetos e o aprimoramento de algumas áreas. Como no caso do gerenciamento de riscos em projetos com utilização de técnicas de simulação, facilitando a administração de outras áreas do conhecimento e minimizando as incertezas nos projetos como prazos e custos. Isso pode ser realizado por meio de softwares simuladores podendo auxiliar os gerentes de projetos com a utilização desses recursos e ferramentas (Carvalho \& Rabechini, 2013).

A Gestão de Projetos deve ser tratada de maneira profissional com a utilização de técnicas e recursos, também deverá conquistar a camada estratégica das empresas. Pois o gerenciamento de portfólio de projetos pode auxiliar nesta missão e uma análise dos surgimentos de novas ideias será possível desenvolver planos estratégicos capaz de competir ou até mesmo melhor que a concorrência, produzindo o despertar nas empresas para sempre inovar e criando projetos cada vez mais complexos, despertando a continuidade por recursos (Carvalho \& Rabechini, 2013). E, por meio desses processos são necessários os auxílios de escritórios de projetos (PMO - Project Management Office) apresentando-se de maneira estratégica e realizando a integração de todos os departamentos que há em uma empresa (Carvalho \& Rabechini, 2013).

Em megaprojetos há um grande número de envolvidos e interessados a serem geridos, devido à complexidade existente (Mok, Shen e Yang, 2015). Os megaprojetos geralmente têm reflexo também na comunidade, gerando impacto ambiental e social (Mok, Shen e Yang, 2015). Dessa forma, a complexidade aumenta também nas relações, conforme a diversidade de perfis existentes para o desenvolvimento do projeto e a precisão da avaliação e o julgamento dos gerentes do projeto frequentemente diminui com o crescimento e complexidade do projeto (Mok, et al. 2015).

Segundo o PMI (2017) o projeto além de cumprir com essas questões básicas, deverá ser elaborado de forma profissional com criatividade e inovação das atividades gerenciais. A elaboração do projeto irá utilizar da aplicação dos cinco grupos de processos e a integração das dez áreas do conhecimento do PMBOK:

Iniciação: serão identificadas as partes interessadas e na área da integração desenvolver o termo de abertura do projeto.

Planejamento: será planejado o gerenciamento do escopo, coletar os requisitos, definir o escopo, criar a EAP (Estrutura Analítica do Projeto), planejar o gerenciamento dos custos, estimar os custos, determinar o orçamento, planejar o gerenciamento da qualidade, planejar o 
gerenciamento dos recursos humanos, planejar o gerenciamento das comunicações, planejar o gerenciamento das partes interessadas, planejar o gerenciamento das aquisições, planejar o gerenciamento do cronograma, definir as atividades, sequenciar as atividades, estimar os recursos das atividades, estimar as durações das atividades, desenvolver o cronograma, planejar o gerenciamento dos riscos, identificar os riscos, realizar a análise qualitativa dos riscos, realizar a análise quantitativa dos riscos, planejar as respostas aos riscos e na área da integração desenvolver o plano de gerenciamento do projeto.

Execução: será mobilizada a equipe do projeto, desenvolver a equipe do projeto, gerenciar a equipe do projeto, conduzir as aquisições, gerenciar o engajamento das partes interessadas, realizar a garantia da qualidade, gerenciar as comunicações e na área da integração orientar e gerenciar a execução do projeto.

Monitoramento e Controle: será validado o escopo, controlar o escopo, controlar o cronograma, controlar a qualidade, controlar as comunicações, controlar os riscos, controlar as aquisições, controlar o engajamento das partes interessadas e realizar o controle integrado de mudanças.

Encerramento: será encerrado o projeto ou fase e encerrado as aquisições.

Além de um excelente gerenciamento de projetos, um dos fatores que contribuem para o sucesso do projeto é a transferência do conhecimento, que minimiza as barreiras que impedem essa transferência entre os colaboradores e de um projeto ao outro (Silva et al., 2015). São algumas as razões que impedem essa transferência: a rotatividade de colaboradores e a não absorção das melhores práticas aprendidas nos projetos (Shatti, Bischff \& Willy, 2017). Apresar dessa constatação, as empresas acabam não agindo de maneira adequada para minimizar essa situação e contribuir com a aquisição de novos conhecimentos (Nonaka \& Toyama, 2003).

\subsection{Gestão do conhecimento}

O conhecimento é fundamental para as organizações (Xu et al., 2006).) O conhecimento é uma mescla de informações, experiências e resolução de especialistas que avaliam e geram novas informações (Davenport e Prusak, 1998). A cada vez que se adquiri conhecimento e se transfere, automaticamente acaba fazendo parte integrante de rotinas, normas e boas práticas beneficiando todos os processos das organizações (Xu et al., 2006). 
O conhecimento pode ser adquirido pelas pessoas ou estar em atividades, produtos e serviços agregando no diferencial dos negócios das organizações (Chuang et al., 2013). Alguns estudos consideram o conhecimento como estratégias organizacionais, mas também não garantindo a sobrevivência em longo prazo das empresas, sendo que algumas formas de conhecimento podem ser relevantes e difíceis de replicar (Donate et al., 2011).

Os tipos de conhecimento são classificados em conhecimento explícito e tácito (Nonaka, 2000); Chiavenato, 2010). Nonaka, (2000), explica como sendo a evolução entre dois tipos de conhecimentos diferentes. O explícito pode ser transmitido às pessoas, documentado em manuais, treinamentos, reuniões, ou seja, ele pode ter acesso por meio de leitura, desenho e vídeos (Chiavenato, 2010). O conhecimento explícito foi conceituado como formal, sistemático, facilmente comunicado e compartilhado por meio de programas de computador, por exemplo (Nonaka, 2000).

No conhecimento tácito não há registro de documentos, tornando-se mais difícil transmitir aos outros, pois está baseado em experiências vividas, ponto de vista de como a pessoa entende determinado fato, reage e pensa sobre a situação em questão (Chiavenato, 2010). Já o conhecimento tácito é altamente pessoal e sua transferência para os outros é árduo (Nonaka, 2000). Segundo Terra e Gordon (2002) o conhecimento tácito é o conhecimento pessoal para fazer seus trabalhos nas organizações, ele é aprendido por períodos de experiências onde o indivíduo desenvolve uma capacidade de execução com sucesso de uma tarefa. Assim, há distinção entre conhecimento tácito e explícito e são quatro padrões básicos de criação de conhecimento em qualquer organização (Nonaka, 2000):

1. De tácito para tácito. Às vezes, certa pessoa compartilha conhecimentos tácitos diretamente com outra pessoa;

2. De explícito para explícito. As pessoas também são capazes de combinar componentes isolados do conhecimento explícito para a constituição de um novo todo;

3. De tácito para explícito. Converter um conhecimento tácito para explícito permitindo seu compartilhamento, por exemplo com uma equipe de desenvolvimento de projeto;

4. De explícito para tácito. Conforme um novo conhecimento explícito é compartilhado em toda organização, outros empregados começam a ampliar, estender e reformular seus próprios conhecimentos tácitos.

$\mathrm{Na}$ empresa criadora de conhecimento esses quatro padrões constituem em uma espécie de espiral de conhecimentos (Nonaka, 2000). 
Atualmente, sobreviver nessa competitividade e constante crescimento no mercado global tem exigido um gerenciamento eficaz do conhecimento organizacional (Xu et al., 2006). Com essa globalização do mercado, o mundo dos negócios tem sofrido grandes mudanças nos últimos anos, na qual essa competitividade atual impactou diversos setores das organizações (Li \& Li, 2000). Portanto, essas mudanças que o mundo vem passando, podemos observar a transformação da sociedade industrial para a sociedade da era da informação ou conhecimento (Lastres \& Albagi, 1999).

Com essas mudanças e competitividade no ambiente econômico, as empresas reconhecem que o gerenciamento do conhecimento é fundamental para o caminho do sucesso nos negócios empresariais (Stewart, 1998). O conhecimento e a informação são matérias primas para gerar riqueza e valor, podendo ser de grande relevância para a produção tradicional, equipamentos e recursos financeiros (Stewart, 1998). O conhecimento é fundamental para a vantagem competitiva de uma empresa (Chen, Yeh \& Huang, 2012).

As organizações atuais administram seus negócios com auxílio da gestão do conhecimento e gerando certo poder de competitividade com sua especialização, conhecimento em um determinado produto/serviço e inovações (Xu et al., 2006). Na visão de Swain \& Lightfoot (2016) a gestão do conhecimento é o processo por meio do qual as empresas geram valor a partir de seus recursos financeiros. Segundo Nonaka (2000) a organização criadora de conhecimento mescla ideias, automaticamente gerando a inovação que é a recriação de determinada visão ou ideal. Desenvolver novos conhecimentos é recriar a empresa e seus profissionais em um processo contínuo de auto renovação pessoal e organizacional (Nonaka, 2000).

O termo em inglês knowledge Management ou gestão do conhecimento é o processo onde os usuários utilizam desse conhecimento adquirido, para serem mais dinâmicos e produtivos nas organizações (Xu et al., 2006). A gestão do conhecimento é um esforço para contribuir no desempenho profissional, organizacional, garantindo que todos tenham acesso ao conhecimento da organização e motivar que compartilhem seus conhecimentos importantes entre os demais colaboradores (Terra \& Gordon, 2002). O objetivo da gestão do conhecimento é desenvolver, ampliar, compartilhar conhecimento entre os gestores e profissionais, ou seja, troca de conhecimento para gerar soluções e boas práticas para tomada de decisão (Chiavenato, 2010). Sistemas de gestão do conhecimento são sistemas de informação desenvolvidos para 
coletar, codificar, integrar e facilitar o conhecimento organizacional em todos os seus processos de negócio (Xu et al., 2006).

Segundo Drucker (2000) a empresa modelo é baseada de conhecimento, composta de especialistas que se reeducam seu desempenho profissional com feedbacks, entre os demais profissionais, gestores e clientes. Portanto, com essas empresas baseadas em informações, houve uma mudança radical de trabalhadores braçais para profissionais do conhecimento (Drucker, 2000). Outro fator que também gera mudança é a economia, para que as empresas invistam em inovações e empreendedorismo, mas a maior mudança vem por parte da tecnologia da informação que fornecem dados e informação para gestão do conhecimento (Drucker, 2000), transformando esse conhecimento em documentos para que sejam absorvidos pelas empresas estruturando seus repositórios (PMI, 2017). Drucker (2000) chegou a falar que a tecnologia em processamento de dados não é fundamental para o desempenho das empresas baseadas em conhecimento, mas ao momento que a tecnologia vai predominando é necessário fazer parte da análise e diagnóstico em informação ou correr o risco de afundarmos nos nossos próprios dados gerados.

Um conceito relacionado a gestão do conhecimento e importante para as organizações é a aprendizagem individual e processos de aprendizagem organizacional, pois tem sido considerada primordial suas medidas do desempenho organizacional da empresa (Qi et al., 2018). A conversão do conhecimento individual em recurso disponível para outras pessoas é a atividade central da empresa criadora de conhecimento (Nonaka, 2000). A capacidade de aprendizagem organizacional e capacidade de gestão do conhecimento são fundamentais para as empresas que fazem negócios na economia baseada no conhecimento (Dayan et al., 2017; Celemín-Pedroche et al., 2017). As organizações reconhecem cada vez mais que o gerenciamento do conhecimento é um dos fatores mais relevantes que contribuem para o sucesso dos negócios (Drucker, 1993). O conhecimento é o principal atributo estratégico e consequentemente o principal meio estratégico é o aprendizado organizacional (Liao et al., 2009). Os novos conhecimentos sempre se originam nas pessoas, pois o conhecimento pessoal de um indivíduo se converteu em conhecimento organizacional valioso para a empresa como um todo (Nonaka, 2000). Portanto, o gerenciamento desse conhecimento é fundamental para o sucesso das empresas. 


\subsection{Sistemas ERP}

A cada dia mais sistemas são desenvolvidos com base em um produto genérico, e logo após, sendo adaptado conforme a necessidade de um cliente (Sommerville, 2011). A era dos primeiros sistemas consistiu com o surgimento do (MRP) Material Requirements Planning, passando pelo (MRP II) Manufacturing Resources Planning e chegando ao (ERP) Enterprise Resource Planning (Stair, 1999). Os sistemas ERP (sistema integrado de gestão empresarial, em inglês enterprise resourse planning), podem ser adaptados para uma empresa, inserindo suas informações de regras e processos de negócio etc (Sommerville, 2011). Os sistemas ERP são sistemas integrados, projetados para oferecer suporte a práticas de negócios, como faturamento, gerenciamento de inventário e encomenda (O’Leary, 2000). O sistema de Enterprise Resource Planning (ERP) pode apoiar toda a fabricação, os pedidos e as atividades de gerenciamento de relacionamento com o cliente, em uma grande empresa (Sommerville, 2011).

Porém, as implementações de ERP podem ser altamente arriscadas e, às vezes, resultando em altos níveis de falha (Ho, Lin, \& Nagalingam, 2009; Sawah, Tharwat, \& Rasmy, 2008). A imprecisão na especificação de requisitos pode causar muitos problemas na implementação do ERP (Sommerville, 2011). Frequentemente, não é a preferência do cliente, onde deve estabelecer novos requisitos, fazer alterações no sistema e consequentemente gerando atrasos de entrega e aumento dos custos (Sommerville, 2011). A especificação dos requisitos funcionais de um sistema deve ser completa e consistente, ou seja, os serviços requeridos pelo usuário devem ser bem definidos e não devem ter definições contraditórias (Sommerville, 2011). Ao elaborar especificações para sistemas complexos é fácil cometer erros, omissões e existem muitos stakeholders (Sommerville, 2011). Os stakeholders têm necessidades diferentes e até inconsistentes, pois os problemas podem surgir após uma análise ou depois de o sistema ter sido entregue ao cliente (Sommerville, 2011).

Há algumas pesquisas sobre a importância do conhecimento dos usuários de ERP para uma implementação de sucesso do ERP, porém, ainda existe um certo obstáculo do ciclo de integração ou transferência de conhecimento (Saide \& Mahendrawathi, 2015). O maior problema na implementação do ERP é a integração de conhecimento tácito e explícito, ou seja, a maior parte desses conhecimentos não está embutida no ERP e sim nos consultores, fornecedores, especialistas e outros stakeholders (Saide \& Mahendrawathi, 2015). 
Para esses sistemas, o processo de configuração envolve a coleta de informações detalhadas sobre negócios do cliente e processos de negócios e a incorporação destes em um banco de dados de configuração. Isso exige, muitas vezes, o conhecimento detalhado da configuração, anotações e ferramentas, e geralmente é realizado por consultores e clientes (Sommerville, 2011). Um sistema ERP genérico inclui uma série de módulos que podem ser compostos de diferentes maneiras para a criação de um sistema para um cliente. O processo de configuração envolve a escolha de quais módulos devem ser incluídos, a configuração desses módulos individuais, a definiçãa de processos de negócios e regras de negócios e definição da estrutura e organização do banco de dados do sistema (Sommerville, 2011).

\section{Metodologia}

O objetivo deste estudo é o de entender, por meio de uma pesquisa qualitativa com triangulação de dados, como o gerenciamento de projetos contribuiu para a implementação e integração de dois sistemas ERP em duas empresas distintas de um mesmo grupo, com o intuito de melhorar a padronização da Gestão do Conhecimento dessas empresas. Segundo Yin (2015), a escolha do método é fundamental para o sucesso da pesquisa científica. O método é o caminho para chegar aos objetivos do estudo (Eisenhardt, 1989). Assim, a pesquisa utilizada para este estudo será de natureza qualitativa e, como método, será aplicado uma pesquisa de campo (Fonseca, 2002), com o uso de entrevistas semiestruturadas. A coleta de dados será realizada por meio de entrevistas semiestruturadas e que não serão a única fonte de dados. Também serão coletados dados secundários das empresas, como exemplo, documentos que fizeram parte do processo de implementação, como também o referencial teórico que dará suporte para esta pesquisa. Assim, será possível realizar a triangulação dos dados (Yin, 2015). Estes dados secundários e o referencial teórico também auxiliarão na elaboração do roteiro de entrevista.

A aplicação da entrevista semiestruturada seguirá alguns cuidados de condução, como por exemplo, a utilização de uma ferramenta para a gravação das mesmas, possibilitando a sua transcrição verbatin (Fielding \& Thomas, 2001). Também serão usadas as notas tomadas durante as entrevistas. Esse procedimento ajudará a minimizar vieses que o pesquisador possa ter no momento de interpretação das gravações nas transcrições (Kvale, 2007). As entrevistas serão realizadas com oito pessoas que estavam envolvidas no projeto: um diretor, um gerente de TI, um chefe de expedição, um supervisor. Mais um gerente administrativo, um coordenador de projetos, um consultor de projetos e um gerente financeiro da empresa desenvolvedora do ERP. Os entrevistados terão os seus nomes mantidos em sigilo por questões éticas. 
O roteiro de entrevista semiestruturada foi elaborado com base no referencial teórico proposto nesse estudo. Ao tomar este cuidado, a intenção é a de estabelecer uma validade (Bianchi \& Ikeda, 2008) para se obter uma relação entre a entrevista e a teoria. O roteiro está dividido em 4 blocos. Sendo que o primeiro bloco tem início com perguntas descritivas do entrevistado, como: Nome do entrevistado; Cargo do entrevistado no momento da implementação do projeto e atual; quantos anos de cargo e de empresa. As questões seguintes, segundo bloco, dizem respeito a Gestão de Projetos: se tem formação na área de Gestão de Projetos; se sempre trabalhou com Gestão de Projetos; se acredita que o uso de Gestão de Projetos para esta implementação foi importante para a realização do mesmo; se em algum momento deixaram de usar a Gestão de Projetos e qual foi o resultado. Já o terceiro bloco está relacionado com questões referentes ao ERP: a implementação e padronização do ERP cumpriu o seu papel dentro das empresas; quais problemas podem surgir durante a implantação de um sistema ERP, em larga escala, em uma organização. O quarto e último bloco está relacionado com a Gestão do Conhecimento: a implementação deste projeto contribuiu na Gestão de Conhecimento das empresas; o quanto a Gestão de Conhecimento é importante para a empresa; especifique esta importância; a Gestão do Conhecimento padronizada permitiu que a empresa se tornasse mais competitiva.

Os dados, após serem coletados, serão analisados com o auxílio do software MAXQDA. Serão realizadas inúmeras leituras das transcrições com o intuito de desenvolver uma estrutura de dados, e posteriormente, serem criadas codificações com o auxílio do software MAXQDA. O uso desse software se justifica por ser mais apropriado para este tipo de pesquisa com dados mais práticos.

\section{Resultados Obtidos e Análise}

Nos próximos tópicos serão apresentadas uma breve descrição das empresas estudadas, a empresa desenvolvedora e responsável pela implementação e integração dos sistemas ERP, unidade de análise e discussões. É importante ressaltar que os resultados e análises foram extraídos de oito entrevistas transcritas em quarenta e duas páginas, oitenta e-mails trocados com a empresa desenvolvedora do ERP e documentação que envolveu o projeto. 


\subsection{Empresas estudadas}

As empresas cujos nomes serão preservados neste relato. A empresa " $\mathrm{X}$ " de natureza privada iniciou suas atividades no dia 02 de maio de 1950 na cidade de São Paulo - SP. Além disso, a empresa atua no ramo de distribuição de papéis e tendo como cultura organizacional uma empresa familiar com sua diretoria já na terceira geração. Em relação à crise no Brasil, que iniciou no segundo semestre de 2014 e alguns assuntos internos, houve a necessidade de abrir outra empresa no mesmo ramo de atividade. A empresa "Y" iniciou suas atividades no dia 05 de setembro de 2016 na cidade de São Paulo - SP. As empresas são consideradas de pequeno porte e, com sua situação financeira não muito estável nos últimos anos, mas conseguindo pagar suas contas em dia.

A taxa de inadimplência está bem próxima do zero por cento. Possui uma estrutura bastante enxuta, contando com vinte e um colaboradores que se dividem em uma hierarquia de Diretoria, Administração, Comercial (Vendas/Compras) e Expedição para o funcionamento de suas atividades. Seus concorrentes são tanto no papel plano (ramo gráfico), como no papel cortado (exemplo papel A4), e seu principal concorrente é a empresa Suzano Papel e Celulose. Atuando há 68 anos no mercado de papéis e suprimentos, contando com os melhores fornecedores do segmento. Nossos clientes são: Gráficas, Editoras, Copistas, Bureaus, Papelaria, Corporativo e Órgãos Públicos.

\subsection{Desenvolvedora do ERP}

A empresa cujo nome será preservado, atua há 20 anos no mercado de desenvolvimento de softwares ERP para o setor de distribuição. A empresa, com sua sede em São Paulo, possuí mais seis filiais e atende mais de trezentos clientes em todos estados Brasileiros. A princípio o ERP já havia sido implantado em maio de 2010 na empresa "X", e com abertura da empresa "Y" em setembro de 2016 houve a necessidade da implantação do mesmo sistema ERP. Entretanto, o problema a ser solucionado foi a unificação das informações das duas empresas para auxiliar os departamentos envolvidos e seus gestores na tomada de decisão e gestão do conhecimento.

\subsection{Unidade de análise}

A princípio o ERP já havia sido implantado em maio de 2010 na empresa "X", e com abertura da empresa "Y" em setembro de 2016 houve a necessidade da implantação do mesmo sistema. Neste caso, seria simples a implantação do mesmo sistema na empresa "Y", pois a 
empresa desenvolvedora do sistema atende mais de 300 clientes com o ERP padronizado para todos. Entretanto, a Diretoria solicitou uma customização do sistema, ou seja, não queria somente informações separadas das duas empresas, mas também informações unificadas em tempo real. Portanto, foi onde surgiu à situação problema, como implementar a integração dos dois softwares ERP? Como unificar as informações das duas empresas para auxiliar os departamentos envolvidos e contribuir com a gestão do conhecimento? O foco está na melhoria, ou seja, desenvolver novas soluções para problemas conhecidos (Gregor e Hevner, 2013).

\subsection{Análises e Discussões}

Com base nas entrevistas realizadas com os stakeholders envolvidos no projeto de implementação e integração do ERP foi possível perceber, com a ajuda do software MAXQDA, as relações entre os principais temas deste relato: Gestão de projetos, Gestão do conhecimento e ERP. Para chegar aos resultados que serão relatados a seguir, foram realizadas inúmeras leituras das entrevistas transcritas, até que fosse possível elencar alguns códigos que emergiram na interpretação dos dados. Esses códigos, além de outros códigos relacionados e identificados nas entrevistas, foram processados no software MAXQDA e se obteve um mapa de correlações (Figura 1) entre os temas que poderão ser visualizados a seguir:

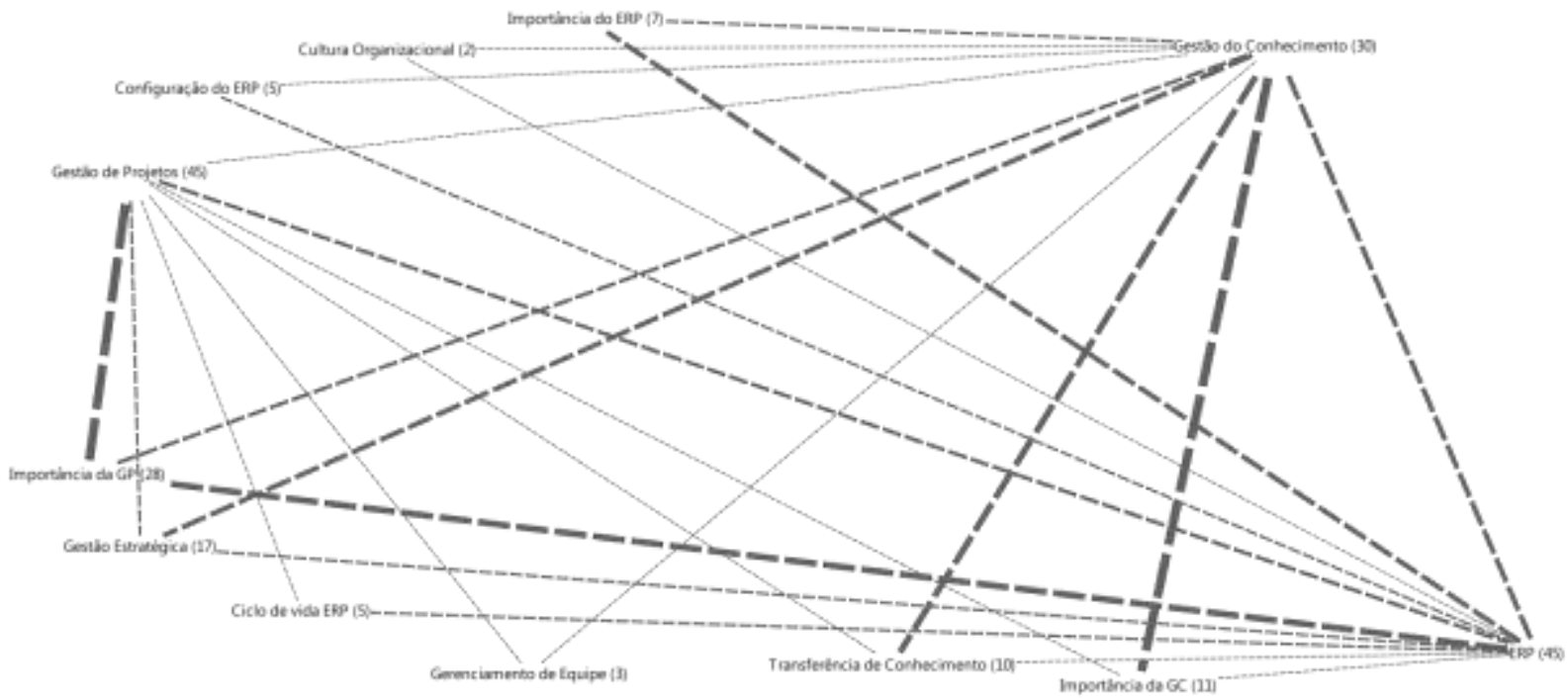

Figura 1: Mapa de correlações entre os temas do estudo.

Fonte: Elaborado pelos autores a partir da análise realizada no Software MAXQDA. 
É importante ressaltar que as linhas mais grossas indicam uma maior intensidade na relação entre os códigos. Já as linhas mais finas indicam uma relação menor. Pode-se perceber, conforme a Figura 1, que os principais temas do estudo foram relacionados com outros temas, como exemplo: a) Gestão de Projetos: Importância da GP, Gestão Estratégica, Ciclo de Vida, Gerenciamento de Equipe, Transferência de Conhecimento, Importância da GC, ERP e Gestão do Conhecimento; b) ERP: Gestão do Conhecimento, Importância do ERP, Cultura Organizacional, Configuração do ERP, Gestão de Projetos, Importância da GP, Gestão Estratégica, Ciclo de Vida ERP, Transferência de Conhecimento e Importância da GC; c) Gestão do Conhecimento: Importância do ERP, Cultura Organizacional, Configuração do ERP, Gestão de projetos, Importância da GP, Gestão Estratégica, Gerenciamento de Equipe, Transferência de conhecimento, Importância da GC e ERP.

Com o intuito de realizar um estudo adequado, além dos dados extraídos das entrevistas (principal fonte do estudo), foram coletados dados por meio de outras fontes, como as informações das empresas estudadas e do projeto pesquisado. Assim, foi possível realizar a triangulação dos dados pesquisados, utilizando também a teoria que dará suporte a essa triangulação. Então, foi possível responder a questão de pesquisa proposta neste estudo: “Como a Gestão de projetos contribuiu para implementação do ERP para melhorar a Gestão do conhecimento dentro das empresas estudada".

Portanto, foram entrevistados oito gestores entre 4 a 32 anos de cargo, apenas dois tem formação na área de Gestão de projetos e um trabalha diariamente com Gestão de projetos. Todos os entrevistados confirmaram que a Gestão de Projetos foi importante para a implementação do ERP. Isso corrobora com o autor Kerzner (2013), que reforça a importância da gestão de projetos em implementações dos projetos. Segundo o entrevistado da empresa desenvolvedora do ERP, que ressaltou não ter deixado de usar a Gestão de projeto em nenhum momento.

"Em nenhum momento, e nossos líderes ficam analisando e acompanhando meio que de longe o andamento do projeto. Eu particularmente nunca deixei de usar a gestão de projetos nas implementações”.

Porém, os entrevistados das distribuidoras comentaram que utilizaram a Gestão de projetos de forma intuitiva, algo não recomendável pelos autores Carvalho \& Rabechini (2013) que afirmam que o gestor de projetos deverá se apresentar de forma profissional, com mais criatividade e sendo menos intuitiva, levando o gerenciamento de projeto como proposta de inovação das atividades gerenciais. Um entrevistado disse: "Sim, só conheço de forma 
intuitiva". Outro: "Na verdade sim, até que dei sorte. Porém, sei que o correto é utilizar a gestão de projetos conforme boas literaturas ensinam de forma mais criativa e inovadora".

A implementação e integração do ERP cumpriu o seu papel dentro das empresas, porque mesmo sabendo da complexidade era necessária a integração das informações de processos de negócios realizados nas duas empresas de distribuição. Sommerville (2007) descreve que o ERP é desenvolvido como um sistema grande, complexo e é adaptado a uma empresa, incorporando informações sobre as regras e os processos de negócios da empresa.

Foram diagnosticados alguns problemas que surgiram durante a implementação do sistema ERP, que foi falta de conexão via remoto, relatórios customizados não funcionavam, falhas na emissão de notas fiscais, imprecisão de requisitos ao duplicar o cadastro de produtos sendo uma empresa certificada FSC (Forest Stewardship Council) e outra não, gerando retrabalho na emissão de notas fiscais. Isso vai ao encontro de afirmações de alguns autores, como exemplo, o Sommerville (2011), diz que a imprecisão na especificação de requisitos pode causar muitos problemas na implementação do ERP.

A implementação deste projeto contribuiu na Gestão de Conhecimento das empresas, pois após dois anos da implementação o cenário é outro, tanto nas empresas como para os seus colaboradores. Nas empresas, porque anteriormente seu faturamento não estava conseguindo pagar as contas e para os colaboradores disponibilizando mais ferramentas e informação gerando novos conhecimentos. Xu (2006) chegou a dizer que os sistemas de informação, a cada dia, são desenvolvidos e implementados para auxiliar no gerenciamento eficiente do conhecimento corporativo. A Gestão do Conhecimento é importante para a empresa, porque ela conseguiu passar pela crise com o planejamento e estratégia, ainda possibilitando abrir outra empresa. A gestão do conhecimento é um esforço para contribuir no desempenho profissional, organizacional, garantindo que todos tenham acesso ao conhecimento da organização e motivar que compartilhem seus conhecimentos importantes entre os demais colaboradores (Terra \& Gordon, 2002). A Gestão do Conhecimento padronizada permitiu que a empresa se tornasse mais competitiva, porque agora são duas empresas no mercado e cada uma focada em determinados clientes, com diversos tipos de produtos. As organizações atuais administram seus negócios com auxílio da gestão do conhecimento e gerando certo poder de competitividade com sua especialização em um determinado produto/serviço e inovações (Xu et al., 2006).

Nas análises, percebe-se que a gestão de projetos tem uma grande relação com a gestão estratégica sendo confirmado por Kerzner (2013) sobre a elaboração de projetos está sendo a 
cada dia mais valorizado nas organizações modernas, principalmente para suas iniciativas de gestão estratégica de negócios. Como comentado por um dos entrevistados: "Eu acredito que a gestão de projetos, ela contribui sim, na gestão estratégica de negócios das empresas... Então, a gestão de projetos ela impacta diretamente na gestão estratégica de negócio com certeza". Outro entrevistado e mais especificamente o Diretor das empresas de distribuição disse:

“Com certeza pode contribuir. A Gestão de Projetos é importante na realidade em todas as áreas, principalmente na área de compras, do estoque, e na área de vendas também é muito importante ter essa Gestão. Quem você vai abordar? Para quem você vai vender? Que região você vai atuar? Com que produtos você vai trabalhar? Então, eu acho bem importante a Gestão de Projetos numa empresa como um todo”.

Algo muito citado nas entrevistas foi à gestão de projetos correlacionando com a importância da gestão do conhecimento e Transferência do conhecimento. Carvalho \& Rabechini, (2013) descreve o uso da integração das áreas do conhecimento em gestão de projetos e o aprimoramento de algumas áreas, facilitando a administração de outras áreas do conhecimento e minimizando as incertezas nos projetos. Chiavenato, (2010) aborda que a importância da gestão do conhecimento é desenvolver, ampliar, compartilhar conhecimento entre os gestores e profissionais, ou seja, troca de conhecimento para gerar soluções e boas práticas. Outra correlação nas entrevistas foi sobre a gestão de projeto que contribui para o ciclo de vida do ERP, inclusive um entrevistado confirma: "Eu acredito que sim, ela pode contribuir para o ciclo de vida do ERP". Outro entrevistado chegou a falar que houve obstáculo na transferência de conhecimento para implementação do ERP.

"Houve obstáculo sim, que é a resistência cultural. A resistência cultural ela é muito evidente na maioria das empresas, por medo, insegurança se aquilo vai dar certo ou não, mas este é o grande obstáculo que a gente enfrenta numa implementação de um ERP. A resistência cultural, mudança de processos, mudança de colaboradores exercendo tal função, isso é, o grande obstáculo que a gente enfrenta e aqui não foi diferente. Há resistência, há um medo da mudança, mas que isso, muita das vezes, atrapalha o próprio crescimento e evolução da empresa. Na nossa empresa, quando a gente finaliza uma implantação numa empresa, a gente chama de passagem de bastão. Onde reunimos toda equipe, e a gente apresenta a empresa para todos os colaboradores. Então, a gente explica quem é? Quem são? O que fazem? Quais são as peculiaridades da empresa? Como é a empresa e se ela tem uma resistência cultural. Como a gente vai dar um 
próximo passo para evoluir este distribuidor, porque este é nosso objetivo e a transferência de conhecimento é no ato que a gente termina uma implantação".

\section{Considerações finais}

Por meio do levantamento e análise dos dados foi possível entender, como o gerenciamento de projetos contribuiu na implementação e integração de dois sistemas ERP em duas empresas distintas de um mesmo grupo, com o intuito de melhorar a padronização da Gestão do Conhecimento dessas empresas. Constatou-se nas análises dos dados a intensidade de relação entre gestão de projetos, gestão do conhecimento e ERP. Estas relações na prática colaboraram para o crescimento e para os processos dos negócios das duas empresas de distribuição de papéis. Outros resultados importantes nas análises envolveram os temas: a importância da gestão de projetos, a importância do ERP, a importância da gestão do conhecimento, gestão estratégica, configuração do ERP, transferência de conhecimento, etc.

Os resultados encontrados neste estudo podem contribuir como exemplos de boas práticas em implementação de sistemas ERP, tanto para os profissionais em Gestão de Projetos, como para Gestores Empresariais. Isso, porque a empresa desenvolvedora do sistema ERP utilizou a todo o momento de forma profissional, a gestão de projetos para a implantação do sistema, atendendo aos requisitos de integração, entrega no prazo estabelecido e dentro do valor orçado. Assim, as duas empresas distribuidoras de papéis conseguiram bons resultados, contribuindo na gestão do conhecimento. Porém, as empresas utilizaram a gestão de projetos de forma intuitiva, consequentemente, surgiram erros como imprecisão na especificação de requisitos como, duplicar o cadastro de produtos, gerando retrabalho por uma das empresas não ser certificada para vender determinados produtos.

Como limitação de pesquisa, pode-se ressaltar a dificuldade em conseguir levantar os documentos do projeto para ajudar na triangulação dos dados, principalmente, pela empresa desenvolvedora do sistema. Outro fato foi a dificuldade em conseguir marcar as entrevistas, pois a maioria dos contatados tiveram problemas em conseguir tempo para dispor à pesquisa. Mas, por fim, foi possível concluir a pesquisa.

A conclusão deste relato direciona para algumas lições aprendidas, a partir desta pesquisa. Passa a ser uma recomendação para outras empresas não somente no setor da distribuição, mas também em outro ramo de atividades. Sugere-se que as empresas com os 
mesmos cenários ao citado nesta pesquisa, utilizem a gestão de projeto de forma profissional nas implantações de sistemas ERP com intuito de contribuir com a gestão do conhecimento organizacional.

\section{Referências}

Bartsch, V.; Ebers, M. \& Maurer, I. (2013). Learning in project-based organizations: The role of project teams' socialcapital for overcoming barriers to learning. International Journal of Project Management, 31(2), 239-251. Carvalho, M. M.\& Rabechini, R. Jr. (2013). Gerenciamento de projetos na prática: casos brasileiros. São Paulo. Atlas.

Crawford, L., Hassner-Nahmias, A.H., 2010. Competências para gerenciar mudanças. Int. J. Proj. Manag. 28 (4), 405-412.

Celemín-Pedroche, M. S., Rubio-Andrada, L., Rodríguez-Antón, J. M. \& Alonso-Almeida, M. D. M. (2017). Causal relationships between organisational learning and performance in the Spanish hotel industry: the managers perception in times of economic crisis. International Journal of Innovation and Learning. 21(3), 274-298.

Chen, Y., Yeh, S., \& Huang, H. (2012). Does knowledge management "fit" matter to business performance? Journal of Knowledge Management, 16(5), 671-687.

Chiavenato, I. (2010). Gestão de pessoas. 3. ed. Rio de Janeiro: Elsevier.

Chuang, S.-H., Liao, C. \& Lin, S. (2013). Determinants of knowledge management with information technology support impact on firm performance. Information Technology and Management. 14(3), 217-230.

Dayan, R., Heisig, P. \& Matos, F. (2017). Knowledge management as a factor for the formulation and implementation of organization strategy. Journal of Knowledge Management. 21(2), 273-298.

Davenport T. \& Prusak L. (1998). Working Knowledge: How Organizations Manage What They Know. Harvard Business School Press: Boston, MA.

Donate, J. M. \& Guadamillas, F. (2011). Organizational factors to support knowledge management and innovation. Journal of Knowledge Management. 15(6), 890-914.

Drucker P. F. (1993). Post-Capitalist Society. Harper \& Collins: New York.

Drucker, P. F. (2000). Gestão do conhecimento / Harvard business review.Tradução Antônio Celso da Cunha Serra. (Cap. 01, pp. 10-11). Rio de Janeiro: Campus.

Eisenhardt, K. M. (1989). Building theories from case study research. Academy of Management Review1, 14(4), 532-550. https://doi.org/10.2307/258557

Fonseca, J. J. S. (2002). Metodologia da pesquisa científica. Fortaleza: UEC. Apostila.

Gregor, S. \& Hevner, A. R. (2013). Positioning and presenting design science research for maximum impact. MIS Quarterly, 37(2), 337-356. Recuperado de http://dl.acm.org/citation.cfm?id=2535658.2535660

Ho, L. T., Lin, G., \& Nagalingam, S. (2009). A risk mitigation framework for integrated enterprise systems implementations for the manufacturing environment. International Journal of Business Information Systems, 4, 209-310.

Kerzner, H., 2013. Gerenciamento de projetos: uma abordagem de sistemas para planejamento, programação e controle, $11^{\text {a }}$ edição. John Wiley \& Sons, Hoboken, N. J.

Kvale, S. (2007). Doing Interviews. Sage Publications, Thousand Oaks.

Lastres, H. M. M. \& Albagi, S. (1999). Informação e globalização na era do conhecimento. Rio de Janeiro: Campus. 
Li H, \& Li L. (2000). Integrating systems concepts into manufacturing information systems. Systems Research and Behavioral Science 17: 135-147.

Liao, S. \& Wu, C. C. (2009). The relationship among knowledge management, organizational learning, and organizational performance. International Journal of Business and Management. 4(4), 64-76.

Martens, C. D. P., Belfort, A. C., Carneiro, K. D. A., \& Martens, M. L. (2014). Gerenciamento de Projetos em Micro e Pequenas Empresas. Revista Pensamento Contemporâneo em Administração (UFF), 8, 151-171.

McKeown, I. \& Philip, G. (2003). Business transformation, information technology and competitive strategies: Learning to fly. International Journal of Information Management. 23(1), 3-24.

Mok, K. Y.; Shen, G. Q. \& Yang, J. (2015). Stakeholder Management studies in mega construction projects: a review and future directions. International Journal of Project Management, 33, 446-457.

Nonaka, I. (2000). Gestão do conhecimento / Harvard business review. Tradução Antônio Celso da Cunha Serra. (Cap. 02, pp. 31). Rio de Janeiro: Campus.

Nonaka, I., \& Toyama, R. (2003). The knowledge-creating theory revisited: knowledge creation as a synthesizing process. Knowledge Management Research \& Practice, 1(1), 2-10.

O’Leary, D. E. (2000). Enterprise Resource Planning Systems: Systems, Life Cycle, Electronic Commerce and Risk. Cambridge, Reino Unido: Cambridge University Press.

PMI, P. M. I. (2017). Um Guia do Conhecimento em Gerenciamento de Projetos (Guia PMBOK) Project Management Institute. (6th ed). EUA: Project Management Institute

Qi, C. \& Chau, P. Y. K. (2018). Will enterprise social networking systems promote knowledge management and organizational learning? An empirical study. Journal of Organizational Computing and Electronic Commerce. 28(1), 31-57.

Saide, \& Mahendrawathi, E.R. (2015). Knowledge Management Support For Enterprise Resource Planning Implementation. 3rd Information Systems International Conference. Procedia Computer Science 72, 613 - 621. Surabaya, Indonesia.

Sawah, S. E., Tharwat, A. A. E. F., \& Rasmy, M. H. (2008). A quantitative model to predict the Egyptian ERP implementation success index. Business Process Management Journal, 14, 288-306.

Shatti, L. A., Bischoff, J. E., \& Willy, C. J. (2017). Investigating the effectiveness of team communication and the balance of member contributions on knowledge acquisition. Knowledge Management Research \& Practice, 16(1), 51-65.

Silva, C. F., Sabbadini, F. S., \& Desidério, M. (2015). Teoria da Criação do Conhecimento Organizacional: o caso da empresa do setor químico. REA - Revista Eletrônica de Administração, 14(1), 120-143.

Souza, C. A.; Vidal, A. G. R. \& Zwicker, R. (2008). Sociedade da informação: os desafios da era da colaboração e da gestão do conhecimento. Organizadores Demerval L. Polizetti; Adalton M. Ozaki. (Cap. 07, pp. 209). São Paulo: Saraiva.

Sommerville, I. (2011). Engenharia de Software. Tradução Ivan Bosnic e Kalinka G. de O. Gonçalves; revisão técnica Kechi Hirama. - 9. ed. - São Paulo: Pearson Prentice Hall.

Stair, R.M.(1999). Princípios de sistemas de informação: uma abordagem gerencial. 2.ed. São Paulo: Editora LTC.

Stewart, T. A. (1998). Capital intelectual: a nova vantagem competitiva das empresas.7. ed. Rio de Janeiro: Campus. 
Swain, D. E., \& Lightfoot, J. E. (2016). A knowledge management framework for global Project development based on Tai Chi principles and practices. International Journal of Managing Projects in Business, 9(3), 624-653.

Tereso, A., Ribeiro, P., Fernandes, G., Loureiro, I., \& Ferreira, M. (2018). Project Management Practices in Private Organizations. Project Management Journal, 50(1), 1-17.

Terra, J. C. C. \& Gordon, C. (2002). Portais corporativos: a revolução na gestão o conhecimento. Tradução Érica Saubermann \& Rodrigo Baroni. (Cap. 03, pp. 57). São Paulo: Negócio Editora.

Teece, D. J., Pisano, G. \& Shuen, A. (1997). Dynamic Capabilities and Strategic Management David. Strategic Management Journal. 18(7), 509-533.

Yin, R. K. (2015). Estudo de Caso: Planejamento e Métodos (5a). Porto Alegre/RS: Editora Bookman.

Xu, L.; Wang, C.; Luo, X. \& Shi, Z. (2006). Integrating knowledge management and ERP in enterprise information systems. Systems Research and Behavioral Science. 23(2),147-156 